\title{
On the Linear-Quadratic, Closed-Loop, No-Memory Nash Game ${ }^{1}$
}

\author{
G. P. PAPAVASSILOPOULOS ${ }^{2}$ AND G. J. OlsDER ${ }^{3}$
}

Communicated by Y. C. Ho

\begin{abstract}
Linear closed-loop no-memory strategies for the LQ Nash game are considered. We exhibit a class of such problems with the property that the solution exists for any finite time interval; for the infinite time case, there exist none or a unique or many solutions, depending on the choice of the parameters. In addition, the limit of the finite time solution as the time interval increases does not have to yield the infinite time case solution. A geometric formulation of the coupled algebraic Riccati equation is given. This formulation seems to be an interesting starting point for a thorough study of these equations.
\end{abstract}

Key Words. Nash games, linear-quadratic games.

\section{Introduction}

The linear-quadratic Nash game has received a lot of attention, but it has not yet reached the degree of maturity of the linear-quadratic control problem. A central issue is the investigation of the linear, closed-loop no-memory strategies. There are several partial results in this direction, but a relatively complete study is not available. See Ref. 1 for a quite recent exposition on this topic and related references.

In this paper, we consider two issues related to this problem. In the first, we consider a class of problems which have the following properties. The finite time version admits a unique solution for any arbitrary but finite

\footnotetext{
${ }^{1}$ This work was supported in part by the United States Air Force, Office of Scientific Research, under Grants Nos. AFOSR-80-0171 and AFOSR-82-0174.

${ }^{2}$ Assistant Professor, Department of Electrical Engineering-Systems, University of Southern California, Los Angeles, California.

${ }^{3}$ Professor, Department of Applied Mathematics, Twente University of Technology, Enschede, Holland.
} 
time interval, whereas the infinite time version might have many, one, or no solution. Moreover, even if the infinite time version has a solution, it does not have to be the limit of the finite time solution. A similar observation was demonstrated in Ref. 2 for a zero-sum, linear-quadratic game. In the second, we provide a geometric formulation of the two algebraic Riccati equations, whose study is a central issue in solving linear-quadratic Nash games. This formulation is of interest and, in our opinion, it deserves further attention.

\section{Linear-Quadratic, Closed-Loop, No-Memory Nash Game}

Consider a dynamic system whose state $x$ evolves according to

$$
\dot{x}=A x+B u_{1}+B u_{2}, \quad x(0)=x_{0}, \quad t \in[0, T],
$$

and two cost functions,

$$
\begin{array}{r}
J_{i}\left(u_{1}, u_{2}\right)=x^{\prime}(T) Q_{i T} x(T)+\int_{0}^{T}\left(x^{\prime} Q_{i} x+u_{i}^{\prime} u_{i}-u_{j}^{\prime} u_{j}\right) d t, \\
i \neq j, i, j=1,2,
\end{array}
$$

$x(t) \in R^{n}, u_{i}(t) \in R^{m}$. The matrices $A, B, Q_{i T}, Q_{i}$ are real, constant, with appropriate dimensions,

$$
Q_{i}=Q_{i}^{\prime} \geq 0, \quad Q_{i T}=Q_{i T}^{\prime} \geq 0,
$$

and $(A, B)$ is assumed to be a controllable pair. Given two strategy spaces $U_{1}, U_{2}$ from which $u_{1}, u_{2}$ are chosen respectively, we call a pair $\left(u_{1}, u_{2}\right) \in$ $U_{1} \times U_{2}$ a Nash equilibrium if

$$
\begin{array}{ll}
J_{1}\left(u_{1}^{*}, u_{2}^{*}\right) \leq J_{1}\left(u_{1}, u_{2}^{*}\right), & \forall u_{1} \in U_{1}, \\
J_{2}\left(u_{1}^{*}, u_{2}^{*}\right) \leq J_{2}\left(u_{1}^{*}, u_{2}\right), & \forall u_{2} \in U_{2} .
\end{array}
$$

We restrict $U_{1}, U_{2}$ to be of the following type:

(i) If $T<+\infty, U_{i}$ consists of all $u_{i}=L_{i} x$, where $L_{i}$ is any $m \times n$ real matrix, which is a continuous function of $t$.

(ii) If $T=+\infty$, in which case we set $Q_{i T}=0, U_{i}$ consists of all $u_{i}=L_{i} x$, where $L_{i}$ is any $m \times n$ real constant matrix.

Our aim is to study the Nash equilibria for cases (i) and (ii) and their interplay.

Case (i): $T<+\infty$. It is known that a pair $\left(u_{1}^{*}, u_{2}^{*}\right)$ is a Nash equilibrium if and only if

$$
u_{i}^{*}=-B^{\prime} K_{i} x, \quad i=1,2,
$$


where

$$
\begin{aligned}
-\dot{K}_{1}= & \left(A-B B^{\prime} K_{2}\right)^{\prime} K_{1}+K_{1}\left(A-B B^{\prime} K_{2}\right)+Q_{1}-K_{2} B B^{\prime} K_{2} \\
& -K_{1} B B^{\prime} K_{1}, \\
-\dot{K}_{2}= & \left(A-B B^{\prime} K_{1}\right)^{\prime} K_{2}+K_{2}\left(A-B B^{\prime} K_{1}\right)+Q_{2}-K_{1} B B^{\prime} K_{1} \\
& -K_{2} B B^{\prime} K_{2}, \\
K_{1}(T)= & Q_{1 T}, \quad K_{2}(T)=Q_{2 T}, \quad t \in[0, T] .
\end{aligned}
$$

Adding and subtracting (5) and (6) gives the following equivalent system:

$$
\begin{aligned}
-(d / d t)\left(K_{1}+K_{2}\right)= & A^{\prime}\left(K_{1}+K_{2}\right)+\left(K_{1}+K_{2}\right) A+Q_{1}+Q_{2} \\
& -\left(K_{1}+K_{2}\right) 2 B B^{\prime}\left(K_{1}+K_{2}\right), \\
K_{1}(T)+K_{2}(T)= & Q_{1 T}+Q_{2 T}, \quad t \in[0, T], \\
-(d / d t)\left(K_{1}-K_{2}\right)= & A^{\prime}\left(K_{1}-K_{2}\right)+\left(K_{1}-K_{2}\right) A+Q_{1}-Q_{2}, \\
K_{1}(T)-K_{2}(T)= & Q_{1 T}-Q_{2 T}, \quad t \in[0, T] .
\end{aligned}
$$

It is obvious now that $K_{1}+K_{2}$ does not blow up in any finite time; and, as $T \rightarrow+\infty, K_{1}(0)+K_{2}(0)$ goes to a stabilizing, positive-semidefinite solution of the corresponding algebraic Riccati equation. Since $(A, B)$ is assumed controllable, if $\left(A,\left(Q_{1}+Q_{2}\right)^{1 / 2}\right)$ is an observable pair this solution of the algebraic Riccati equation is unique. $K_{1}-K_{2}$ obeys the linear differential equation (10), and thus does not blow up in any finite time. As $T \rightarrow$ $+\infty, K_{1}(0)-K_{2}(0)$ might become unbounded, or oscillate, or converge to some limit; what will happen depends obviously on $A, Q_{1}-Q_{2}$ and $Q_{1 T}-$ $Q_{2 T}$. Our conclusion is the following: The finite time Nash game (1)-(3) admits a unique linear solution for any arbitrarily large, but finite, time interval $[0, T]$. We can choose $A, Q_{i}, Q_{i T}$ so as to have that $K_{1}, K_{2}$ converge, or become unbounded, or oscillate.

Case (ii): $\mathbf{T}=+\infty$. If a Nash equilibrium exists, it will be of the form

$$
u_{i}^{*}=-B^{\prime} K_{i} x, \quad i=1,2,
$$

where

$$
\begin{aligned}
& 0=\left(A-B B^{\prime} K_{2}\right)^{\prime} K_{1}+K_{1}\left(A-B B^{\prime} K_{2}\right)+Q_{1}-K_{2} B B^{\prime} K_{2}-K_{1} B B^{\prime} K_{1}, \\
& 0=\left(A-B B^{\prime} K_{1}\right)^{\prime} K_{2}+K_{2}\left(A-B B^{\prime} K_{1}\right)+Q_{2}-K_{1} B B^{\prime} K_{1}-K_{2} B B^{\prime} K_{2} .
\end{aligned}
$$


Adding and subtracting (13) and (14) yields the following equivalent system:

$$
\begin{aligned}
& 0=A^{\prime}\left(K_{1}+K_{2}\right)+\left(K_{1}+K_{2}\right) A+Q_{1}+Q_{2}-\left(K_{1}+K_{2}\right) 2 B B^{\prime}\left(K_{1}+K_{2}\right), \\
& 0=A^{\prime}\left(K_{1}-K_{2}\right)+\left(K_{1}-K_{2}\right) A+Q_{1}-Q_{2} .
\end{aligned}
$$

Since

$$
J_{1}^{*}+J_{2}^{*}=\int_{0}^{+\infty} x^{\prime}\left(Q_{1}+Q_{2}\right) x=x_{0}^{\prime}\left(K_{1}+K_{2}\right) x_{0}, Q_{1}+Q_{2} \geq 0,
$$

$K_{1}+K_{2}$ has to be positive semidefinite. Thus, assuming $(A, B)$ controllable, and $\left(A,\left(Q_{1}+Q_{2}\right)^{1 / 2}\right)$ observable, we conclude that $K_{1}+K_{2}$ will be the unique positive-definite stabilizing solution of (15). For $K_{1}-K_{2}$ to exist, we have to assume that there exists a $Y=Y^{\prime}$ satisfying

$$
0=A^{\prime} Y+Y A+Q_{1}-Q_{2}
$$

in which case

$$
Y=K_{1}-K_{2} \text {. }
$$

It is obvious that (17) might have many, one, or no solutions. Even if (17) has a unique solution, this solution does not have to be the limit of the solution of the differential equation (10)-(11), since the solution of (10)(11) might not have a limit as $T \rightarrow+\infty$. Of course, if the solution of $(10)-(11)$ has a limit as $T \rightarrow+\infty$, then this limit is a solution of (17). The basic conclusion is the following. The system (13)-(14) might have one, or many, or no solutions. Even if it has a solution, this solution does not need to be the limit of the solutions of $(5)-(7)$.

That the limit of the finite time solution, as $T \rightarrow+\infty$, does not have to be the infinite-time solution has also been observed in Ref. 2 for the case of zero-sum games.

Although the above-stated conclusion refers to the Riccati equations, which provide necessary but not sufficient conditions for a Nash equilibrium pair, we can use that to create Nash games where the finite-time solution does not converge as $T \rightarrow+\infty$ to the infinite-time solution.

\section{Geometric Formulation of the Coupled Algebraic Riccati Equations}

It is known that the problem of finding all the symmetric solutions of the algebraic Riccati equation

$$
0=A^{\prime} K+K A+Q-K B B^{\prime} K
$$


can be reformulated equivalently as follows (see Refs. 3 and 4): (18) can be written as

$$
\left[\begin{array}{l:l}
K & -I
\end{array}\right]\left[\begin{array}{c:c}
A & -B B^{\prime} \\
\hdashline-Q & -A^{\prime}
\end{array}\right]\left[\begin{array}{c}
I \\
K
\end{array}\right]=0
$$

or

$$
A_{s}\left[\begin{array}{c}
I \\
K
\end{array}\right]=\left[\begin{array}{c}
I \\
K
\end{array}\right] X
$$

where $X$ is some $n \times n$ matrix and $A_{s}$ is the system matrix

$$
A_{s}=\left[\begin{array}{rr}
A & -B B^{\prime} \\
-Q & -A^{\prime}
\end{array}\right]
$$

Thus, if one is able to find the $n$-dimensional subspaces $\mathscr{V}$ in $R^{2 n}$ for which it holds that

$$
A_{s} \mathscr{V} \subseteq \mathscr{V}
$$

or the $2 n \times n$ matrices

$$
\left.V=\left[-V_{1}\right]\right\} n
$$

whose columns constitute a basis for $\mathscr{V}$ and $V_{1}^{-1}$, exist and $V_{2} V_{1}^{-1}$ is symmetric, one has a solution

$$
K=V_{2} V_{1}^{-1}
$$

of (18). A simple-minded way of doing that is briefly the following: If

$$
A V=V Y \text {, }
$$

for some $n \times n$ matrix $Y$, let $T^{-1} Y T$ be the Jordan form of $Y$, and thus

$$
A_{s} V T=V T T^{-1} Y T \text {. }
$$

Let

$$
\begin{aligned}
& V T=\bar{V}=\left[\begin{array}{llll}
v_{1} & v_{2} & \cdots & v_{n}
\end{array}\right], \\
& T^{-1} Y T=\left[\begin{array}{cccc}
\lambda & 1 & 0 & \cdots \\
0 & \lambda & 1 & \\
0 & 0 & \lambda & \\
& & & \ddots
\end{array}\right] .
\end{aligned}
$$


Then,

$$
\begin{aligned}
& A_{s} v_{1}=\lambda v_{1}, \\
& A_{s} v_{2}=\lambda v_{2}+v_{1}, \\
& \ldots,
\end{aligned}
$$

Thus, we can find the eigenvectors and generalized eigenvectors of $A_{s}$, and group them appropriately so as to create

$$
\bar{V}=\left[\begin{array}{c}
\bar{V} \\
\bar{V}_{2}
\end{array}\right]
$$

Since

$$
V_{1}=\bar{V}_{1} T^{-1}, \quad V_{2}=\bar{V}_{2} T^{-1},
$$

we need $\bar{V}_{1}$ to be invertible and

$$
V_{2} V_{1}^{-1}=\bar{V}_{2} \bar{V}_{1}^{-1}
$$

to be symmetric. Thus, the whole issue is the appropriate choice of $n$ eigenvectors and generalized eigenvectors of $A_{s}$. Needless to say that we are usually interested only in some of the solutions of (18), so that the closed-loop matrix $A-B B^{\prime} K$ is stable. The point to be made here is that the generic problem that underlies this method of solving (18) is the problem of finding the $n$-dimensional subspaces $\mathscr{V}$ in $\mathbb{R}^{2 n}$ which are $A_{s}$ invariant, i.e.,

$$
A_{s} \mathscr{V} \subseteq \mathscr{V} \text {. }
$$

Let us now generalize the previous formulation to the linear-quadratic, closed-loop, no-memory Nash game. Let the system equation and the two costs be given by

$$
\begin{aligned}
& \dot{x}=A x+B_{1} u_{1}+B_{2} u_{2}, \\
& J_{i}=\int_{0}^{\infty}\left(x^{\prime} Q_{i} x+u_{i}^{\prime} u_{i}+u_{j}^{\prime} R_{i j} u_{j}\right) d t, \quad i \neq j, i, j=1,2,
\end{aligned}
$$

where $Q_{i}, R_{i j}$ are symmetric. If a pair $\left(u_{1}, u_{2}\right)$ is a Nash equilibrium where each $u_{i}$ at time $t$ is a linear function of $x(t)$, it has to be given by

$$
u_{i}=-B_{i}^{\prime} K_{i} x, \quad i=1,2,
$$

where the matrices $K_{1}, K_{2}$ are symmetric and satisfy the system

$$
\begin{aligned}
0= & K_{1}\left(A-B_{2} B_{2}^{\prime} K_{2}\right)+\left(A-B_{2} B_{2}^{\prime} K_{2}\right)^{\prime} K_{1}+Q_{1}+K_{2} B_{2} R_{12} B_{2}^{\prime} K_{2} \\
& -K_{1} B_{1} B_{1}^{\prime} K_{1},
\end{aligned}
$$




$$
\begin{aligned}
0= & K_{2}\left(A-B_{1} B_{1}^{\prime} K_{1}\right)+\left(A-B_{1} B_{1}^{\prime} K_{1}\right)^{\prime} K_{2}+Q_{2}+K_{1} B_{1} R_{21} B_{1}^{\prime} K_{1} \\
& -K_{2} B_{2} B_{2}^{\prime} K_{2} .
\end{aligned}
$$

Conditions (30) and (31) are only necessary for the pair (29) to be a Nash equilibrium.

This system can be written as

$$
\begin{gathered}
{\left[I, K_{1}, K_{2}\right]\left[\begin{array}{ccc}
Q_{1} & A^{\prime} & 0 \\
A & -B_{1} B_{1}^{\prime} & -B_{2} B_{2}^{\prime} \\
0 & -B_{2} B_{2}^{\prime} & B_{2} R_{12} B_{2}^{\prime}
\end{array}\right]\left[\begin{array}{c}
I \\
K_{1} \\
K_{2}
\end{array}\right]=0} \\
{\left[I, K_{1}, K_{2}\right]\left[\begin{array}{ccc}
Q_{2} & 0 & A^{\prime} \\
0 & B_{1} R_{21} B_{1}^{\prime} & -B_{1} B_{1}^{\prime} \\
A & -B_{1} B_{1}^{\prime} & -B_{2} B_{2}^{\prime}
\end{array}\right]\left[\begin{array}{c}
I \\
K_{1} \\
K_{2}
\end{array}\right]=0}
\end{gathered}
$$

or equivalently,

$$
\begin{aligned}
& {\left[\begin{array}{ccc}
Q_{1} & A^{\prime} & 0 \\
A & -B_{1} B_{1}^{\prime} & -B_{2} B_{2}^{\prime} \\
0 & -B_{2} B_{2}^{\prime} & B_{2} R_{12} B_{2}^{\prime}
\end{array}\right]\left[\begin{array}{c}
I \\
K_{1} \\
K_{2}
\end{array}\right]=\left[\begin{array}{cc}
-K_{1} & -K_{2} \\
I & 0 \\
0 & I
\end{array}\right]\left[\begin{array}{c}
X \\
Z
\end{array}\right],} \\
& {\left[\begin{array}{ccc}
Q_{2} & 0 & A^{\prime} \\
0 & B_{1} R_{21} B_{1}^{\prime} & -B_{1} B_{1}^{\prime} \\
A & -B_{1} B_{1}^{\prime} & -B_{2} B_{2}^{\prime}
\end{array}\right]\left[\begin{array}{c}
I \\
K_{1} \\
K_{2}
\end{array}\right]=\left[\begin{array}{cc}
-K_{1} & -K_{2} \\
I & 0 \\
0 & I
\end{array}\right]\left[\begin{array}{c}
Y \\
W
\end{array}\right],}
\end{aligned}
$$

where $X, Y, Z, W$ are some $n \times n$ matrices. It is easy to see that

$$
X=W
$$

We can put these two conditions together to obtain

$$
A_{s}^{N}\left[\begin{array}{c}
I \\
K_{1} \\
K_{2}
\end{array}\right]=\left[\begin{array}{ccc}
I & 0 & 0 \\
0 & I & 0 \\
0 & 0 & I \\
K_{1} & 0 & K_{2} \\
K_{2} & K_{1} & 0
\end{array}\right]\left[\begin{array}{c}
X \\
Y \\
Z
\end{array}\right]
$$

where

$$
A_{s}^{N}=\left[\begin{array}{ccc}
A & -B_{1} B_{1}^{\prime} & -B_{2} B_{2}^{\prime} \\
0 & B_{1} R_{21} B_{1}^{\prime} & -B_{1} B_{1}^{\prime} \\
0 & -B_{2} B_{2}^{\prime} & B_{2} R_{12} B_{2}^{\prime} \\
-Q_{1} & -A^{\prime} & 0 \\
-Q_{2} & 0 & -A^{\prime}
\end{array}\right]
$$


Let

$$
L_{1}=\left[\begin{array}{ccc}
I & 0 & 0 \\
0 & 0 & 0 \\
0 & 0 & 0 \\
0 & I & 0 \\
0 & 0 & I
\end{array}\right], \quad L_{2}=\left[\begin{array}{lll}
0 & 0 & 0 \\
I & 0 & 0 \\
0 & 0 & 0 \\
0 & 0 & 0 \\
0 & I & 0
\end{array}\right], \quad L_{3}=\left[\begin{array}{lll}
0 & 0 & 0 \\
0 & 0 & 0 \\
I & 0 & 0 \\
0 & 0 & I \\
0 & 0 & 0
\end{array}\right] .
$$

Solving (36) is easily seen to be equivalent to the following problem: Find the $n$-dimensional subspaces $M$ of $R^{3 n}$ for which it holds that

$$
A_{s}^{N} \mathcal{M} \subseteq L_{1} \mathscr{M}+L_{2} \mathcal{M}+L_{3} \mathcal{M} \text {. }
$$

If the $3 n \times n$ matrix

$$
\left.M=\left[\begin{array}{c}
M_{0} \\
\hdashline \bar{M}_{1} \\
\hdashline \bar{M}_{2}
\end{array}\right]\right\} n
$$

is a basis for $\mathcal{M}$, where $M_{0}^{-1}$ exists and $M_{1} M_{0}^{-1}, M_{2} M_{0}^{-1}$ are symmetric, then

$$
K_{1}=M_{0} M_{0}^{-1}, \quad K_{2}=M_{2} M_{0}^{-1}
$$

solve (30), (31). Solving (39) for $\mathscr{M}$ is equivalent to finding the matrices $M$ for which it holds that

$$
A_{s}^{N} M=L_{1} M X_{1}+L_{2} M Y_{1}+L_{3} M Z_{1},
$$

for some $n \times n$ matrices $X_{1}, Y_{1}, Z_{1}$. If we were to imitate (24)-(28), we should proceed as follows. Let $T$ be an $n \times n$ invertible matrix. Then, (40) can be written as

$$
A_{s}^{N} \bar{M}=L_{1} \bar{M} T^{-1} X_{1} T+L_{2} \bar{M} T^{-1} Y_{1} T+L_{3} \bar{M} T^{-1} Z_{1} T,
$$

where

$$
\bar{M}=\left[\begin{array}{l}
M_{0} T \\
\frac{M}{M} \frac{T}{T} \\
\bar{M}_{2} \frac{T}{T}
\end{array}\right] .
$$

We can assume that $T^{-1} X_{1} T$ is in Jordan form as we did for $T^{-1} Y T$ in (27), but the form of $T^{-1} Y_{1} T, T^{-1} Z_{1} T$ is not necessarily Jordan. Thus, a difficulty of imitating (24)-(28) lies in not being able, with a single matrix $T$, to have Jordan forms $T^{-1} X_{1} T, T^{-1} Y_{1} T, T^{-1} Z_{1} T$ simultaneously for the three matrices $X_{1}, Y_{1}, Z_{1}$. 
A different geometric formulation of $(30)-(31)$ is the following: Let

$$
\begin{aligned}
& A-B_{1} B_{1}^{\prime} K_{1}-B_{2} B_{2}^{\prime} K_{2}=X, \\
& B_{2} B_{2}^{\prime} K_{1}-B_{2} R_{12} B_{2}^{\prime} K_{2}=-Z, \\
& B_{1} B_{1}^{\prime} K_{2}-B_{1} R_{21} B_{1}^{\prime} K_{1}=-Y .
\end{aligned}
$$

We can rewrite (30)-(31) as

$$
\begin{aligned}
& K_{1} X=-\left(A^{\prime} / 2\right) K_{1}-Q_{1} / 2+W_{1}, \\
& K_{2} X=-\left(A^{\prime} / 2\right) K_{2}-Q_{2} / 2+W_{2}, \\
& \left(A^{\prime} / 2\right) K_{1}+Q_{1} / 2+W_{1}=-K_{2} Z, \\
& \left(A^{\prime} / 2\right) K_{2}+Q_{2} / 2+W_{2}=-K_{1} Y,
\end{aligned}
$$

for some $W_{1}, W_{2}$. Thus, we can write the following system which is equivalent to (30)-(31):

$$
\begin{aligned}
& {\left[\begin{array}{ccc}
A & -B_{1} B_{1}^{\prime} & -B_{2} B_{2}^{\prime} \\
-Q_{1} / 2 & -A^{\prime} / 2 & 0 \\
-Q_{2} / 2 & 0 & -A^{\prime} / 2
\end{array}\right]\left[\begin{array}{c}
I \\
K_{1} \\
K_{2}
\end{array}\right]+\left[\begin{array}{c}
0 \\
W_{1} \\
W_{2}
\end{array}\right]=\left[\begin{array}{c}
I \\
K_{1} \\
K_{2}
\end{array}\right] X,} \\
& {\left[\begin{array}{ccc}
0 & -B_{2} B_{2}^{\prime} & B_{2} R_{12} B_{2}^{\prime} \\
0 & 0 & 0 \\
-Q_{1} / 2 & -A^{\prime} / 2 & 0
\end{array}\right]\left[\begin{array}{c}
I \\
K_{1} \\
K_{2}
\end{array}\right]+\left[\begin{array}{c}
0 \\
W_{3} \\
-W_{1}
\end{array}\right]=\left[\begin{array}{c}
I \\
K_{1} \\
K_{2}
\end{array}\right] Z,} \\
& {\left[\begin{array}{ccc}
0 & B_{1} R_{21} B_{1}^{\prime} & -B_{1} B_{1}^{\prime} \\
-Q_{2} / 2 & 0 & -A^{\prime} / 2 \\
0 & 0 & 0
\end{array}\right]\left[\begin{array}{c}
I \\
K_{1} \\
K_{2}
\end{array}\right]+\left[\begin{array}{c}
0 \\
-W_{2} \\
W_{4}
\end{array}\right]=\left[\begin{array}{c}
I \\
K_{1} \\
K_{2}
\end{array}\right] Y,}
\end{aligned}
$$

for some $X, Y, Z, W_{1}, W_{2}, W_{3}, W_{4}$.

If we denote by $\mathcal{N}$ the space in $R^{7 n}$ spanned by the columns of the matrix

$$
\left[\begin{array}{c}
I \\
K_{1} \\
K_{2} \\
W_{1} \\
W_{2} \\
W_{3} \\
W_{4}
\end{array}\right],
$$


we can rewrite (41)-(43) as

$$
A_{1} \mathcal{N} \subseteq L \mathcal{N}, \quad A_{2} \mathcal{N} \subseteq L \mathcal{N}, \quad A_{3} \mathcal{N} \subseteq L \mathcal{N},
$$

where $A_{1}, A_{2}, A_{3}$ are known $3 n \times 7 n$ matrices and

$$
L=\left[\begin{array}{lllllll}
I & 0 & 0 & 0 & 0 & 0 & 0 \\
0 & I & 0 & 0 & 0 & 0 & 0 \\
0 & 0 & I & 0 & 0 & 0 & 0
\end{array}\right]
$$

Thus, we have imbedded the problem (30)-(31) into (44), i.e., into finding the $n$-dimensional spaces $\mathcal{N}$ in $R^{7 n}$ which satisfy simultaneously the three invariance properties

$$
A_{i} \mathcal{N} \subseteq L \mathcal{N}, \quad i=1,2,3 .
$$

In concluding this section, we should point out two things. The first one is that we need to solve (39) or (44) which are generalizations of (22). Although we do not know at present how to achieve that, it seems to us that these formulations of the problem of solving (30)-(31) are quite appealing and deserve further attention. The second is that the role of the system matrix $A_{s}$ [see (21)] of the control problem is assumed in the game case by $A_{s}^{N}$ [see (37)] in conjunction with $L_{1}, L_{2}, L_{3}$ [see (38)].

\section{References}

1. BASAR, T., and Olsder, G. J., Dynamic Noncooperative Game Theory, Academic Press, New York, New York, 1982.

2. MAGEIRoU, E., Values and Strategies for Infinite-Time, Linear-Quadratic Games, IEEE Transactions on Automatic Control, Vol. AC-21, pp. 547-550, 1976.

3. PotTer, J. E., Matrix Quadratic Solutions, SIAM Journal on Applied Mathematics, Vol. 14, pp. 496-501, 1966.

4. MARTENSSON, K., On the Matrix Riccati Equations, Information Sciences, Vol. 3, pp. 17-49, 1971. 